\title{
Clever new method coaxes chromatin from living tissues
}

\section{BY MARISSA FESSENDEN}

18 MARCH 2015

Natalia Sinjushina \& Evgeniy Meyke/Shutterstock.com Small samples: A new method allows researchers to study chromatin in tissue during different stages of development.

Several autism-linked genes affect the structure of chromatin - the coiled complex of DNA and proteins that helps to regulate gene expression. Extracting chromatin from tissues, such as brain, is technically challenging. As a result, researchers studying chromatin typically take this material from cultured cells, but these cells may not yield accurate answers to important gene expression questions because they show different patterns of gene expression than do cells in living tissue.

Scientists have created a way to study chromatin in tissues by adding a few preparatory steps to a method called ChIP-Seq. The modified method, described 2 February in Cold Spring Harbor Protocols, allows researchers to extract chromatin from fresh or frozen tissue samples weighing as little as 1 milligram ${ }^{1}$.

The first step entails carefully separating the tissue into smaller chunks that react more readily with the chemicals needed for chromatin removal. Then, after researchers fix the chromatin in place within the cells' nuclei, they bathe the fixed tissue in a solution that causes the cellular material surrounding the nuclei to swell. (The protein scaffold outside the cells balloons in tandem.) Finally, the swollen material is stripped away, leaving behind the intact nuclei. 


\section{Spectrum | Autism Research News}

https://www.spectrumnews.org

Researchers can then extract chromatin from the nuclei and prepare it for sequencing using a version of ChIP-Seq. A few tweaks optimize the process for small amounts of chromatin, as the tiny tissue samples typically available for analysis contain far fewer cells than can be obtained from culture.

The scientists used the method to extract chromatin from the budding limbs of mouse and human embryos, revealing proteins involved in limb development. Examining how the structure of chromatin in specific tissues changes throughout development could shed light on the origins of complex diseases such as autism, they say.

The scientists also include advice for specific tissues. For example, brain tissue may require higher concentrations of certain chemicals to break apart cells and free the nuclei.

\section{References:}

1. Cotney J.L. and J.P. Noonan Cold Spring Harb. Protoc. Epub ahead of print (2015) PubMed

Cite this article: https://doi.org/10.53053/PMFC5417 